\section{Map reveals} global Zika risk

More than 2.17 billion people around the world live in habitats suitable for the mosquito-borne Zika virus, which is currently spreading in Central and South America.

Women infected with Zika virus when pregnant are at increased risk of giving birth to infants with microcephaly, which stunts brain development. Jane Messina at the University of Oxford, UK, and her colleagues used data on virus incubation, mosquito ranges and environmental and socio-economic variables associated with Zika outbreaks to produce a fine-scale global map that predicts areas with a high risk of virus spread (pictured, in red).

The map shows Florida and Texas to be ideal environments for Zika transmission, whereas southern Argentina, Uruguay and Chile, for example, are less likely to experience outbreaks. More than 5 million births in the Americas alone could be at risk from Zika infection over the next year, the authors say. elife http://doi.org/bfmg (2016)

\section{PHYSICS}

\section{Cold coffee beans grind smaller}

Roasted coffee beans that are ground when cold give smaller particles than those ground at room temperature, which could affect the drink's flavour.

Christopher Hendon at the Massachusetts Institute of

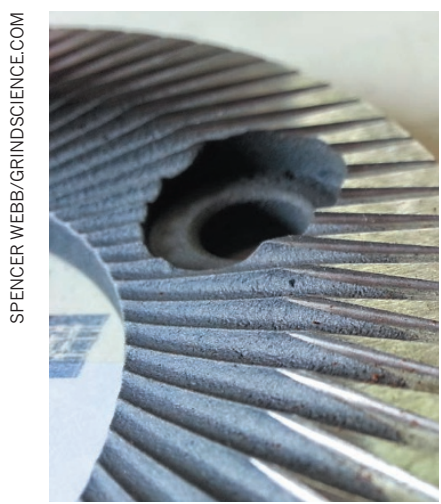

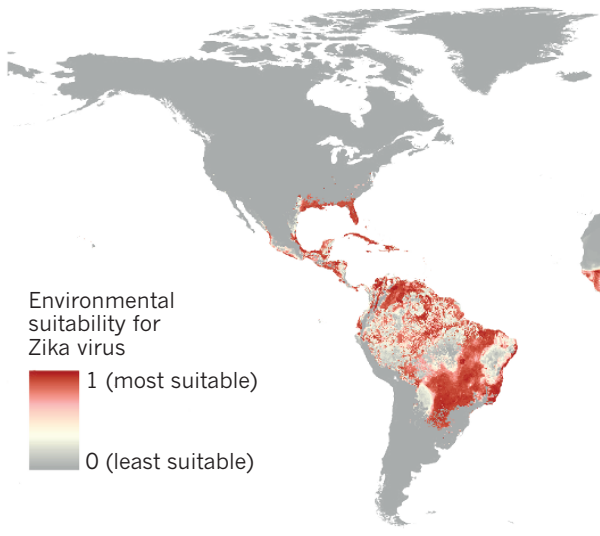

Technology in Cambridge and his colleagues ground coffee beans (grinding burr pictured) and measured particle size, comparing beans from four countries and also those from a single source that were kept at four different temperatures before grinding. They found that particle-size distribution was similar across beans from different parts of the world. But particles became smaller and more uniform in size as the temperature dropped, with the largest change occurring between room temperature and $-19^{\circ} \mathrm{C}$.

Smaller and more uniformly sized coffee particles could release more flavour during brewing and might allow more coffee to be brewed from the same amount of grounds, the authors suggest.

Sci. Rep. 6, 24483 (2016)

\section{GENETICS}

\section{'Wellderly' secrets revealed}

A key to healthy ageing could be genetic protection against cognitive decline.

Eric Topol and Ali Torkamani of the Scripps Translational Science Institute in La Jolla, California, and their colleagues sequenced and analysed the genomes of more than 500 disease-free people over the age of 80 . They found that these 'wellderly' individuals had a lower genetic risk of Alzheimer's disease and heart disease than a group of adults representing the general population, but about the same genetic risk of diabetes and cancer.
The authors suggest that wellderly people are genetically distinct from people who reach extreme old age by surviving significant health challenges. However, they add that the study was small and needs replication. Those in the wellderly group tended to be highly educated - a factor known to be linked to long lifespans, perhaps because of healthier lifestyles and diets. Cell http://dx.doi.org/10.1016/j. cell.2016.03.022 (2016)

\section{METABOLISM}

\section{New hormone regulates glucose}

Researchers have discovered a hormone that modulates the rapid release of glucose and insulin into the bloodstream in between meals.

Atul Chopra at Baylor

College of Medicine in

Houston, Texas, and his colleagues found that levels of the protein hormone - which they called asprosin - peaked in the blood during fasting in humans and mice, and is made by white fat tissue. Giving asprosin to mice boosted their blood glucose and insulin levels. The hormone bound to liver cells, triggering glucose release.

Mice and humans with insulin resistance (prediabetes) showed elevated levels of asprosin. Treating insulin-resistant mice with an asprosin-blocking antibody lowered their blood insulin levels. Decreasing asprosin could be a way to treat type 2 diabetes, the authors say. Cell 165, 566-579 (2016)

\section{How old age limits adaptability}

Altered activity in two brain regions could explain why older animals struggle to adapt to changes in their environment.

Previous research has shown that a circuit connecting the thalamus and striatum helps animals to adapt their previous learning to a change in conditions. Jesus BertranGonzalez at the University of Queensland in Brisbane, Australia, and his colleagues found that old mice showed weaker connections from the thalamus to the striatum, and had altered electrical properties in certain striatal neurons, compared with young mice. The team trained mice to press one lever to receive a preferred food and another for a non-preferred food; when researchers reversed these associations, old mice, as well as young mice with damage to this circuit, had trouble adapting their actions to the new rules.

The authors suggest that defects in this pathway impair an ageing animal's ability to integrate new and existing information when deciding how to act.

Neuron http://dx.doi.org/ 10.1016/j.neuron.2016.03.006 (2016)

\section{$\rightarrow$ NATURE.COM}

For the latest research published by Naturevisit:

www.nature.com/latestresearch 